\title{
Planetary Nebulae in the Scheme of Binary Evolution
}

\author{
Noam Soker \\ Dept. of Physics, University of Haifa-Oranim, Tivon 36006, Israel
}

\begin{abstract}
In this review I present the binary model for the shaping of planetary nebulae (PNe) as I view it, in the context of historical evolution of other models for the shaping of $\mathrm{PNe}$ over more than 30 years. In describing the binary model, I concentrate on works published since the last IAU meeting on PNe. I think stellar companions are behind the shaping of bipolar PNe, i.e., having two lobes with an equatorial waist between them, and extreme elliptical PNe, e.g., having a dense equatorial ring, but no lobes. The question of whether a planet is required to spin the progenitor in order to form a moderate elliptical $\mathrm{PN}$, or whether single stars can form moderate elliptical PNe, I consider to be an open question.
\end{abstract}

\section{Introduction}

The rich variety of planetary nebula $(\mathrm{PN})$ shapes have attracted attention for a long time. Different mechanisms were discussed to form the axisymmetrical structures of PNe. Magnetic fields, via different mechanisms, were, and continue to be, quite popular. The idea that the galactic magnetic field shapes $\mathrm{PNe}$ was raised several times (e.g., Grinin \& Zvereva 1968), but was always 'killed', recently by Corradi, Aznar, \& Mampaso (1998). Internal magnetic fields, via several different mechanisms, have been discussed for more than 40 years (e.g., Gurzadyan 1962; Woyk 1968 [note his drawings!]; Pascoli 1985, 1997; Chevalier \& Luo 1994; García-Segura 1997; García-Segura et al. 1999; García-Segura, \& López 2000; García-Segura, López, \& Franco 2001; Matt et al. 2000; Blackman et al. 2001; Gardiner, \& Frank 2001; see also review by García-Segura in these proceedings). These models assume that the magnetic field comes from the AGB progenitor, and attribute dynamical effects to the magnetic field, i.e., the magnetic pressure and/or tension become comparable to that of the thermal pressure. Some magnetic effects were criticized during these years (e.g., Menzel 1968; Soker \& Zoabi 2002 and references therein). In other models, the magnetic field has a secondary role. In several papers (e.g., Soker 1998b, 2000, 2001b ; Soker, \& Clayton 1999; Soker \& Zoabi 2002) I propose that the large-scale stellar magnetic field is strong enough for the formation of magnetic cool spots on the AGB stellar surface. The spots may regulate dust formation, hence mass loss rate, leading to axisymmetric mass loss and the formation of elliptical PNe. Despite its role in forming cool spots, the large scale magnetic field is too weak to play a dynamic role and directly influence the wind from the AGB star, as required by the dynamic models. 
A popular mechanism for shaping $\mathrm{PNe}$ is the so called interacting winds model (see review by Frank 1999). The basic model assumes mass concentration in the equatorial plane from the AGB phase. Later, the fast wind from the central star of the PN accelerates the nebula, with higher velocities and distances attended in the polar directions, forming elliptical or bipolar PNe. Ionization plays a significant role in the evolution (Mellema 1995; Mellema \& Frank 1995). The idea of mass concentration in the equatorial plane is old; Khromov \& Kohoutek (1968) suggested that mass concentration in the equatorial plane can focus the ionization radiation to the poles. However, it became a popular idea with the seminal work of Balick (1987). Interaction of the fast and slow winds definitely occurs in $\mathrm{PNe}$, and is successful in explaining some properties of $\mathrm{PNe}$. However, it cannot explain all properties in many of the observed bipolar PNe, e.g., the high momentum and kinetic energy observed in several bipolar $\mathrm{PNe}$ and proto-PNe (Bujarrabal et al. 2001). Other problems are mention by Frank (2000; also Balick 2000).

Other, less popular, mechanisms were proposed in the literature, e.g., a circumstellar disk that survived from the pre-main sequence phase (e.g., Kastner et al. 1996).

My view (Soker \& Harpaz 1992; Soker 1998b; Soker \& Zoabi 2002) is that a stellar binary companion is required to spin up the AGB star in order for the later to have a strong magnetic field. The equatorial mass concentration required for the interacting wind model to form bipolar and extreme elliptical PNe also seems to require a stellar binary companion (e.g., Soker \& Livio 1989; Iben \& Livio 1993), e.g., as obtained in the simulations of Mastrodemos \& Morris (1998, 1999). The companion, then, may cause other effects, e.g., accreting mass, forming an accretion disk (Mastrodemos \& Morris 1999), and blowing jets (Morris 1987; Soker \& Rappaport 2000).

Before further exploring binary models, I clarify some terms.

\section{Claryfing Some Terms}

- Single stars. Some mechanisms may work for single stars, e.g., fast rotation (García-Segura et al. 1999; but not their claim for critical slow rotation; see Glatzel 1998). However, in these cases, I argue (Soker \& Harpaz 1999; Soker 2001b ) the envelope posses more angular momentum than a single AGB star can maintain from its main sequence phase. Hence the envelope must be spun-up by a stellar companion. I would refer to these cases as binary evolution, rather than single star evolution, although the process itself involves only one star.

- Magnetic field. There are some indications for magnetic fields around AGB stars and in PNe, mainly from maser emission (e.g., Miranda et al. 2001). This does not necessarily support models with dynamical effects of magnetic fields in the AGB progenitors. There can be other sources for the magnetic field. (1) The magnetic field can be localized in specific regions. This is predicted if there is a higher mass loss rate from active regions, e.g., higher mass loss rate from cool spots (e.g., Soker \& Zoabi 2002). (2) An accreting companion, whether a WD or a main sequence 
star, will amplify the magnetic field. Hence the strong field may result from an accreting companion. (3) The magnetic field may result from a magnetically active main sequence companion. Accreting main sequence companions will be spun-up, and may become magnetically active (Jeffries \& Stevens 1996; Soker \& Kastner 2002).

- Morphology. I will use the following 4 main axisymmetrical morphology classes. (i) Bipolar PNe: PNe having two lobes with and equatorial waist between them. (ii) Extreme elliptical PNe: PNe having a large equatorial concentration of mass, e.g., a ring, but no, or only small, lobes. They may possess jets. (iii) Moderate elliptical PNe: PNe having a large scale elliptical shape, with a shallow density variation from equator to poles. They may possess jets. (iv) Spherical PNe: PNe whose entire structure show a general circular shape. If there are even small parts which are axisymmetric rather than spherical, the PN is not spherical (I will not discuss these any more here; for some properties of spherical PNe see Soker $[2002 a])$.

\section{Binary systems and PN Structures}

The idea that binary systems are behind the axisymmetrical structure of $\mathrm{PNe}$ is old, and was mentioned many times in the literature, more times than can be mentioned here; a few examples are Fabian \& Hansen (1979), Phillips, \& Reay (1983), Miranda (1995), Kolesnik \& Pilyugin (1986), and Pollacco \& Bell (1997). In particular, Morris (1981, 1987, 1990; Mastrodemos \& Morris 1999) and Livio (1982, 1993, 1995, 1997, 2000; Livio, Salzman, \& Shaviv 1979; Iben \& Livio 1993; Livio \& Pringle 1996) were pushing the idea of binary interaction for a long time, proposing many ideas and models. In my research on the connection between binary systems and PNe, I benefited from their works, as well as from observational works by Corradi and Schwarz (e.g., Corradi 1995; Corradi \& Schwarz 1995; Corradi et al. 1999, 2001; Schwarz et al. 1997), and Bond (e.g., Bond 2000). In their works, Corradi and Schwarz nailed down the similarity between symbiotic nebulae, which known to be formed by binary systems, and bipolar PNe (for other, some earlier, papers on this connection and binarity see Mammano \& Ciatti 1975; Cohen et al. 1985; Morris 1990; Goodrich 1991; Lee \& Park 1999).

Main supporting observations for the shaping of some PNe by binary systems is in Soker (1997; as well as different processes caused by a companion), and supporting observations for binary shaping of bipolar PNe are in Soker (1998a; see table 1 there), and references in these papers. I only list several of them here, in addition to new results (for the explanation of massive progenitor of bipolar PNe see §5). (i) 16 PNe with central binary systems, one of them bipolar and the rest extreme elliptical PNe, are known (Bond 2000). (ii) Other PNe and proto-PNe show strong indication for binarity (e.g., Rodríguez, Corradi, \& Mampaso 2001; the 120 years side to side variation in M2-9, Doyle et al. 2000). (iii) The similar morphology of bipolar PNe and many symbiotic nebulae. (iv) Expansion velocities in many bipolar PNe proto-PNe are much higher than the escape velocity from AGB stars. (v) Statistically, binary systems which avoid common envelope can account for bipolar PNe (Soker \& Rappaport 2000), as 
models requires (next section). Common envelope systems can account for a large fraction of elliptical PNe (Yungelson, Tutukov, \& Livio 1993; Han, Podsiadlowski, \& Eggleton 1995). However, it seems that binary stellar systems cannot account for all non spherical PNe; either single stars can form moderate elliptical, or planets are required.

\section{Current Status of Binary Models}

I summarize now my view on the role plaid by stellar companions, as appeared in several of my papers from the last 5 years. As is well known, accreting companions can blow jets (Livio 2000), or if not well collimated, termed collimated fast wind (CFW; Soker \& Rappaport 2000). The CFW (or jets) can shape the nebula in several ways. If the CFW is strong, it will form two lobes (Morris 1987), and form bipolar PNe. A close companion can enhance the equatorial density by spining-up the AGB star (Soker \& Rappaport 2000) and/or focusing the AGB wind (Mastrodemos \& Morris 1999). Like in symbiotic nebulae, the companion should be outside the AGB envelope, at least during part of the evolution. I therefore argue (Soker 1998a), that most bipolar PNe are formed from companions outside the envelope; in a few cases (e.g., NGC 2346) the companion can enter the envelope at a late stage. The different routes that can lead to the rich variety of bipolar shapes is summarized in Soker (2002b ). Stellar companions at large orbital separations (tens of AU; see Soker 2001a ) may blow weak CFW, which will form extreme elliptical PNe, possibly with small lobes. Most extreme elliptical $\mathrm{PNe}$ are formed via common envelope evolution (e.g., Bond \& Livio 1990).

Binary systems can lead to other effects. Examples are displacement from axisymmetry (Soker \& Rappaport 2001; Soker \& Hadar 2002), formation of a circumbinary disk (e.g., Van Winckel 1999; Jura, Chen \& Plavchan 2002), and leading to backflowing material in the post-AGB phase (Soker 2001c ).

It is not clear, though, whether binary systems are behind the multiplerings found by $\mathrm{HST}$ in several PNe and proto-PNe (e.g., Hrivnak, Kwok, \& Su 2001), directly, by their gravity and orbital motion, or indirectly, by spiningup the AGB star (for different views, see Soker 2002c and Y. Simis in these proceedings).

Because stellar binary companions cannot account for all non-spherical PNe, either single stars can form moderate elliptical PNe, or, as I tend to think, planets are required to spin-up these AGB stars (Soker 2001b). Whereas I am confident that binary companions shape bipolar and extreme elliptical $\mathrm{PNe}$, whether a companion is required to shape moderate elliptical PNe, I consider an open question.

\section{Massive Progenitors of Bipolar PNe}

Although more than three years have past since I published my explanation for the observations that bipolar PNe are formed from more massive stars, annoyingly, this correlation is still, wrongly, used as contradictory to binary models. I presented the explanation of Soker (1998a; described below, but see Soker 1998a 
for detail) in my talk; despite that I got 2 questions after the talk regarding that same point. Because of that, I devote a section just for this observation.

As noted by many surveys (e.g., Zuckerman \& Gatley 1988), bipolar PNe are concentrated toward the Galactic plane. This and composition differences from elliptical PNe strongly suggest that progenitors of bipolar PNe are more massive than those of the other PNe (e.g., Greig 1972; Acker 1980; Kaler 1983; Torres-Peimbert \& Peimbert 1997). As explained in Soker (1998a) the main differences between massive $\left(M \gtrsim 2 M_{\odot}\right.$ on the main sequence) and low-mass progenitors are that the radii of the massive progenitors become much larger on the AGB than on the RGB, and their envelope mass is much larger than that of low-mass progenitors. Because of their large radius on the RGB, low mass stars interact with a companion, that could have form bipolar PN, already on their RGB track. Either they lose their envelope and never form a PN, because of their low mass envelope, or the companion spirals-in to collide with their core, after which the star evolves as rapidly rotating single star, hence forming an elliptical PNe.

Another, more simple, explanation is that more massive stars are more likely than low mass stars to harbor close massive companions that lead to the formation of bipolar PN. This was suggested for binary systems that go through a stable Roche lobe overflow (Soker \& Livio 1994).

I end with a comment I made during a debate conducted at the Asymmetrical Planetary Nebulae II (APN II) meeting: There is a class of systems called massive X-ray binaries, with a neutron star and a massive companion. So all of them contain massive stars; does this mean that these binary systems are 'single stars'?

\section{Future Works and Predictions}

Theory. In order to reproduce bipolar morphologies, there is a need to perform 3D gasdynamical simulations where both stars blow wind simultaneously: The AGB blows a slow wind, either spherically symmetric or concentrated to the equatorial plane, while the compact companion, a WD or a main sequence star, blows a CFW (collimated fast wind, or jets). Since the binary are close, and the nebula is large, and the CFW is more than an order of magnitude faster than the slow wind, these are very demanding simulations.

Observations. The main issue is to detect binary companions. Some methods with a table of 16 PNe with known central binary systems are given by Bond (2000, and references therein). Careful spectroscopic analysis can lead to more detection, as was done recently by Rodríguez et al. (2001). X-ray observations can also lead to detection of companions. An accreting WD companion (Livio \& Shaviv 1975) will be too faint to be distinguish from the bright central stars of PNe. However, spun-up main sequence stars can become magnetically active, and emit strongly in X-ray. This was suggested to be the case for the point-like X-ray emission in the PNe NGC 6543 and NGC 7293 (Guerrero et al. 2001). Some magnetically active stars exist around WDs (e.g., Jeffries \& Stevens 1996; Bond et al. 2001). The main sequence companions were spun-up by accreting from the AGB progenitor, and then became magnetically active (Jeffries \& Stevens 1996; Soker \& Kastner 2002). Therefore, I think that X-ray 
observations may reveal the existence of main sequence companions to central stars of PNe (Soker \& Kastner 2002).

Acknowledgments. I thank Guillermo García-Segura for stimulating my research by giving a good scientific fight back, with his high quality scientific work, and by bringing different arguments for single star models during our lively and friendly discussions.

\section{References}

Acker, A. 1980, A\&A, 89, 33

Balick, B. 1987, AJ, 94, 671

Balick, B. 2000, in Asymmetrical Planetary Nebulae II: From Origins to Microstructures,

Astronomical Society of the Pacific Conference Series, Vol. 199,

eds. J. Kastner, N. Soker, \& S. Rappaport, 41

Blackman, E. G., Frank, A., Markiel, J. A., Thomas, J. H., \& Van Horn, H. M. 2001, Nature, 409, 485

Bond, H. 2000, in Asymmetrical Planetary Nebulae II: From Origins to Microstructures, Astronomical Society of the Pacific Conference Series, Vol. 199,

eds. J. Kastner, N. Soker, \& S. Rappaport, 115

Bond, H., \& Livio, M. 1990, ApJ, 355, 568

Bond, H. E., Mullan, D. J., O'Brien, M., S., \& Sion, E. M. 2001, ApJ, 560, 919

Bujarrabal, V., Castro-Carrizo, A., Alcolea, J., \& Sánchez Contreras, C. 2001, A\&A, 377,868

Chevalier, R. A., \& Luo, D. 1994, ApJ, 421, 225

Cohen, M., Dopita, M. A., Schwartz, R. D., \& Tielens, A. G. G. M. 1985, AJ, 297, 702

Corradi, R. L. M. 1995, MNRAS, 276, 521

Corradi, R. L. M.; Aznar, R., \& Mampaso, A. 1998, MNRAS, 297, 617

Corradi, R. L. M., Ferrer, O. E., Schwarz, H. E., Brandi, E., \& García, L. 1999, A\&A, 348,978

Corradi, R. L. M., Livio, M., Balick, B., Munari, U., \& Schwarz, H. E. 2001, ApJ, 553, 211

Corradi, R. L. M., \& Schwarz, H. E. 1995, A\&A, 293, 871

Doyle, S., Balick, B., Corradi, R. L. M., \& Schwarz, H. E. 2000, AJ, 119, 1339

Fabian, A. C., \& Hansen, C. J. 1979, MNRAS, 187, 283

Frank, A 1999, NewAR, 43, 31

Frank, A. 2000, in ASP Conf. Ser. 199, Asymmetrical Planetar Nebulae II: From Origins to Microstructures, ed. J. H. Kastner, N. Soker, \& S. Rappaport (San Francisco: ASP), 225

García-Segura, G. 1997, ApJ, 489, L189

García-Segura, G., Langer, N., Rozyczka, M., \& Franco, J. 1999, ApJ, 517, 767

García-Segura, G., \& López, J. A. 2000, ApJ, 544, 336

García-Segura, G., López, J. A., \& Franco, J. 2001, ApJ, 560, 928

Gardiner, T. A., \& Frank, A 2001, ApJ, 557, 250

Glatzel, W. 1998, A\&A, 339, L5

Goodrich, R. W. 1991, ApJ, 366, 163

Greig, W. E. 1972, A\&A, 18, 70 
Grinin, V. P., \& Zvereva, A. M. 1968, Planetary Nebulae, eds. Donald E. Osterbrock \& Charles Robert O'Dell, IAU Sym. no. 34, (D. Reidel Pub.; Dordrecht), 287

Guerrero, M. A., Chu, Y.-H., Gruendl, R. A., Williams, R. M., Kaler, J. B. 2001, ApJ, $553, \mathrm{~L} 55$

Gurzadian, G. A. 1962 Planetary Nebulae (Moscow; English translation: 1969, Gordon and Breach Sc. Pub., Ney York)

Han, Z., Podsiadlowski, P., \& Eggleton, P. P. 1995, MNRAS, 272, 800

Hrivnak, B. J., Kwok, S., \& Su, K. Y. L. 2001, AJ, 121, 2775

Iben, I., Jr., \& Livio, M. 1993, PASP, 105, 1373

Jeffries, R. D., \& Stevens, I. R. 1996, MNRAS, 279, 180

Jura, M., Chen, C., \& Plavchan P. 2002, preprint.

Kaler, J. B. 1983, ApJ, 271, 188

Kastner, J. H., Weintraub, D. A., Gatley, I., Merrill, K. M., \& Probst, R. G. 1996, ApJ, 462,777

Khromov, G. S., \& Kohoutek, L. 1968, Planetary Nebulae, eds. Donald E. Osterbrock \& Charles Robert O'Dell, IAU Sym. no. 34, (D. Reidel Pub.; Dordrecht), 227

Kolesnik, I. G., \& Pilyugin, L. S. 1986, AZh, 63, 279

Lee, H.-W., Park, M.-G. 1999, ApJ, 515, L89

Livio, M. 1982, A\&A, 105, 37

Livio, M. 1993, in Planetary Nebulae, ed. R. Weinberger \& A. Acker (Dordrecht: Kluwer), 279

Livio, M. 1995, in Asymmetrical Planetary Nebulae, ed. A. Harpaz \& N. Soker (Ann. Israel Phys. Soc., 11) (Bristol: IOP), 51

Livio, M. 1997, Planetary Nebulae, IAU Sym. 180, ed. H. J. Habing \& H. J. G. L. M. Lamers (Dordrecht: Kluwer), 74

Livio, M. 2000, in ASP Conf. Ser. 199, Asymmetrical Planetary Nebulae II: From Origins to Microstructures, ed. J. H. Kastner, N. Soker, \& S. Rappaport (San Francisco: ASP), 243

Livio, M., \& Pringle, J. E. 1996, ApJ, 465, L55

Livio, M., Salzman, J., \& Shaviv, G. 1979, MNRAS, 188, 1

Livio, M., \& Shaviv, G. 1975, Nature, 258, 308

Mammano, A., \& Ciatti, F. 1975, A\&A, 39, 405

Mastrodemos, N., \& Morris, M. 1998, ApJ, 497, 303

Mastrodemos, N., \& Morris, M. 1999, ApJ, 523, 357

Matt, S., Balick, B., Winglee, R., \& Goodson, A. 2000, ApJ, 545, 965

Mellema, G., 1995, MNRAS, 277, 173

Mellema, G., \& Frank, A. 1995, MNRAS, 273, 401

Menzel, D. H. 1968, Planetary Nebulae, eds. Donald E. Osterbrock \& Charles Robert O'Dell, IAU Sym. no. 34, (D. Reidel Pub.; Dordrecht), 279

Miranda, L. F. 1995, A\&A, 304, 531

Miranda, L. F., Gómez, Y., Anglada, G., \& Torrelles, J. M. 2001, Nature, 414, 284

Morris, M. 1981, ApJ, 249, 572

Morris, M. 1987, PASP, 99, 1115

Morris, M. 1990, in From Miras to Planetary Nebulae: Which Path for Stellar Evolution?, ed. M. O. Mennessier \& A. Omont (Paris: Editions Frontires), 520

Pascoli, G. 1985, A\&A, 147, 257

Pascoli, G. 1997, ApJ, 489, 946 
Phillips, J. P., \& Reay, N. K. 1983, A\&A, 117, 33

Pollacco, D. L., \& Bell, S. A. 1997, MNRAS, 284, 32

Rodrguez, M., Corradi, R. L. M., \& Mampaso, A. 2001, A\&A, 377, 1042

Schwarz, H. E., Aspin, C., Corradi, R. L. M., \& Reipurth, B. 1997, A\&A, 319, 267

Soker, N. 1997, ApJS, 112, 487

Soker, N. 1998a, ApJ, 496, 833

Soker, N. 1998b, MNRAS, 299, 1242

Soker, N. 2000, ApJ, 540. 436

Soker, N. 2001a, ApJ, 558, 157

Soker, N. 2001b, MNRAS, 324, 699

Soker, N. 2001c, MNRAS, 328, 1081

Soker, N. 2002a (astro-ph/0105142).

Soker, N. 2002b, MNRAS, in press (astro-ph/0107554)

Soker, N. 2002c, ApJ, in press (astro-ph/0110474)

Soker, N., \& Clayton, G. C. 1999, MNRAS, 307, 993

Soker, N., \& Hadar, R. 2002, MNRAS, in press.

Soker, N., \& Harpaz, A. 1992, PASP, 104, 923

Soker, N., \& Harpaz, A. 1999, MNRAS, 310, 1158

Soker, N., \& Kastner 2002, ApJ, in press (astro-ph/0111266)

Soker, N., \& Livio, M. 1989, ApJ, 339, 268

Soker, N., \& Livio, M. 1994, ApJ, 421, 219

Soker, N., \& Rappaport, S. 2000, ApJ, 538, 241

Soker, N., \& Rappaport, S. 2001, ApJ, 557, 256

Soker, N., \& Zoabi, E. 2002, MNRAS, 329, 204

Torres-Peimbert, S., \& Peimbert, M. 1997, Planetary Nebulae, IAU Sym. 180, ed. H. J. Habing \& H. J. G. L. M. Lamers (Dordrecht: Kluwer), 74

Van Winckel, H. 1999, in Asymptotic Giant Branch Stars, eds. T. Le Bertre, A. Lebre, \& C. Waelkens, ASP Conference Series (San Francisco), p. 465

Woyk, E. 1968, Planetary Nebulae, eds. Donald E. Osterbrock \& Charles Robert O'Dell, IAU Sym. no. 34, (D. Reidel Pub.; Dordrecht), 275

Yungelson, L. R., Tutukov, A. V., \& Livio, M. 1993, ApJ, 418, 794 F

Zuckerman, B., \& Gatley, I. 1988, ApJ, 324, 501 